Paidéia, 2004, 14 (28), 169 -176

\title{
A DEPRESSÃO NO TESTE DAS PIRÂMIDES COLORIDAS DE PFISTER ${ }^{1}$
}

\author{
Anna Elisa de Villemor Amaral ${ }^{2}$ \\ Ricardo Primi \\ Flávia Helena Zanetti Farah \\ Sandra Mônica da Silva \\ Lucila Moraes Cardoso \\ Renata da Rocha Campos Franco \\ Universidade São Francisco
}

\begin{abstract}
Resumo: O objetivo desse estudo foi investigar o desempenho de pacientes com depressão no teste das Pirâmides Coloridas de Pfister. Foram investigados 19 pacientes com depressão, previamente diagnosticados pela SCID, e seus desempenhos foram comparados com os resultados obtidos com uma amostra de 110 indivíduos não pacientes que participaram de um estudo normativo. Adotou-se como procedimento a versão do Pfister de 24 matizes e a realização de três pirâmides bonitas. Os resultados apontaram para um significativo aumento do verde acompanhado da constância absoluta da cor violeta, bem como a maior incidência de pirâmides cortadas e de formações tendendo a estruturas. A combinação desses indicadores possibilita identificar pacientes com pânico havendo 86,4\% de sensibilidade. Tais resultados permitem afirmar que o teste de Pfister pode colaborar para o diagnóstico de depressão, sendo contudo necessários outros recursos para maior segurança diagnóstica.
\end{abstract}

Palavras-chave: teste das pirâmides coloridas de Pfister; avaliação psicológica; depressão.

\section{THE DEPRESSION ON THE PFISTER'S COLORS PYRAMIDS TEST}

Abstract: In this study our purpose was to identify the aspects in the Color Pyramid Test of Pfister which may be useful to the assessment of depressive patients. Our sample was composed by 19 participants previously assessed by the SCID as depressives. We used the Pfister's version composed by 24 colors and only the three beautiful pyramids. The results are compared with the performance of 110 no-patients and demonstrates that the high frequency of green, the absolute constancy of violet, the stratified forms with cuts or tendency to structures are significant for the group of patients Our results indicates that the sensibility of Pfister for the diagnosis of depression based on this aspects is about $86,4 \%$. This test can helps satisfactory for the assessment of depression when associated to others techniques. This study contributes to the necessity to verify the possibilities and limitations in the use of this test.

Key-words: color pyramid test of Pfister; psychological assessment; depression.

O teste das Pirâmides Coloridas de Pfister é um instrumento usado em diagnósticos há mais de 50 anos, tanto em pesquisas quanto na clínica, tendo uma boa aceitação pelos profissionais do campo da psicologia. Entretanto, trabalhos relativos à psicopatologia são raros (Villemor Amaral, 1978) e

\footnotetext{
${ }^{1}$ Artigo recebido para publicação em 30/10/2003; aceito em 22/04/2004. ${ }^{2}$ Endereço para correspondência: Anna Elisa de Villemor Amaral, Programa de Estudos Pós-Graduados em Psicologia, Universidade São Francisco, Rua Alexandre Rodrigues Barbosa, 45, Itatiba, SP, Cep 13251900, E-mail: anna.villemor@saofrancisco.edu.br
}

por meio de uma verificação bibliográfica constatase que muito poucas publicações foram feitas recentemente. Encontram-se alguns trabalhos relacionados às aplicações dessa técnica nos diversos campos da psicologia (Bauer, 1979.) principalmente na clínica e na área da saúde mental (Carnio \& Loureiro, 1993; Oliveira, Pasian \& Jacquemin, 2001), mas, praticamente, inexistem pesquisas que visem à validação desse instrumento para diagnóstico diferencial. Por se tratar de um instrumento não verbal e rela- 


\section{Anna Elisa de Villemor Amaral}

tivamente lúdico, não exigindo habilidades específicas, o teste de Pfister é bastante útil quando se trata de apreender a dinâmica afetiva do paciente, principalmente no caso de indivíduos com dificuldade de expressão verbal ou gráfica.

O significado afetivo das cores é algo reconhecido amplamente na cultura e as noções sobre as relações entre cores e afetos sempre estiveram presentes no simbolismo das manifestações artísticas, rituais religiosos, bem como no senso comum. Há, no entanto, muito a ser pesquisado com vistas a comprovar cientificamente essas noções. Desde que Rorschach desenvolveu seu método psicodiagnóstico, atribuindo valor muito importante para as respostas de cor como representantes da afetividade (Rorschach, 1972), descobriu-se o sentido inegável do estímulo cromático no campo das emoções, faltando ainda compreender e verificar de modo mais sistemático as possíveis relações entre diferentes cores e diferentes afetos.

No senso comum as cores tem um significado bastante marcado, como é o caso, por exemplo, da cor preta que simboliza, na cultura ocidental, luto, morte e tristeza, remetendo, inevitavelmente, aos sentimentos presentes na depressão. Já em relação às cores vivas, estas lembram energia e alegria.

Do ponto de vista científico, há pesquisas que estabelecem correlações entre os aspectos sensoriais e os estados depressivos, fazendo referências à cor e a sensorialidade. Estevão (1997), em seu estudo sobre a depressão, afirma tratar-se de um estado de luto muito primitivo, manifestado sem culpa e caracterizado por uma lentificação e insensibilidade da sensorialidade, acompanhada por apatia, tristeza e sensações de impotência e desesperança. Para esse autor, mesmo a intensidade das cores esmaece, assim como o claro-escuro, dando lugar a uma tonalidade cinza, sem contrastes. Os cheiros param de ser percebidos, as texturas deixam de ser registradas, os sons ficam amortecidos e podem até desaparecer. Os processos fisiológicos se alteram e os movimentos corporais ficam mais lentos.

Do ponto de vista epidemiológico, verificase que é cada vez maior o número de pessoas deprimidas em todo o mundo. Segundo a Organização Mundial da Saúde, 10 a 15 \% da população mundial sofrerá de depressão no decorrer da vida (Gauer,
1998). Há alguns fatores que podem explicar esse aumento como, por exemplo, a exigência do mundo de hoje que cultiva apenas a produção e a eficácia, esquecendo o ser humano (Berlinck \& Fedida, 2000); alterações das condições de vida dos indivíduos que impõem esforços enormes a cada pessoa (Nuber, 1991) e o contato com a dura realidade que faz com que o indivíduo, para se proteger desse contato tão frustrante e ameaçador, desenvolva a depressão como recurso de autodefesa (Estevão, 1997).

Lima (1999), apoiado em pesquisas de base populacional em epidemiologia psiquiátrica, constatou que os índices de depressão maior e distimia, bem como outros transtornos depressivos, são altos independentes do lugar onde a pesquisa foi conduzida, do tipo de instrumento diagnóstico u sado e dos períodos de tempo para os quais a prevalência se aplica. Afirma ainda que a depressão é mais comum entre mulheres, pessoas divorciadas ou separadas, que vivem sozinhas, que possuem baixo nível de escolaridade e renda, desempregados e moradores de zonas urbanas.

Numa revisão da literatura sobre a depressão e seus sintomas encontram-se diversos pontos de vista. Para Smith (1999), os sintomas da depressão abrangem aspectos afetivos, motivacionais, cognitivos e vegetativos, sendo descritos de forma consistentemente semelhante há mais de dois mil anos.

O DSM-IV insere a depressão na seção relativa aos transtornos de humor e traz os seguintes critérios para o diagnóstico de episódio depressivo: humor deprimido, perda do interesse e prazer pelas coisas e ainda pelo menos cinco sintomas de uma lista de sete, que estendem-se por um período de duas semanas, a saber: a) perda ou ganho de peso; b) alterações no sono; c) agitação ou retardo psicomotor; d) fadiga; e) sentimento de inutilidade ou culpa; f) baixa capacidade de concentração; g) indecisão; h) pensamentos de morte e ideação suicida. Esses sintomas devem causar sofrimento clinicamente significativo ou prejuízo no funcionamento social ou ocupacional do indivíduo, não tendo origem em efeitos fisiológicos diretos de uma substância ou condição médica, também não podendo ser melhor explicados por luto - perda ou morte de um ente querido.

O limite entre a depressão clínica e as 
flutuações normais de humor não é claro e mesmo a presença ou ausência de sintomas pode ser discutida. Para Lima (1999) as investigações quanto ao diagnóstico de co-morbidade costumam ser imprecisas e na prática clínica a depressão e a ansiedade freqüentemente coexistem. Para Rodrigues (2000) a "depressão como categoria diagnóstica, que responderia a uma causalidade única, não existe” (p.155).

Segundo Nuber (1991), há quatro sinais característicos típicos da depressão: uma pessoa deprimida não consegue alegrar-se com mais nada, não alimenta mais esperança, não acredita mais em si mesma, nem nos outros, e é incapaz de tomar decisões, o que faz com que ela encare as causas de resultados negativos como duradouros, abrangentes e pessoais, enquanto que as causas dos resultados positivos são vistos como passageiros, específicos e externos. Essa maneira pessimista de enfrentar a vida não faz sentido do ponto de vista da realidade circundante.

A depressão pode se apresentar com diferentes facetas e em intensidades muito variadas (Cunha, Prieb, Goulart \& Lemes, 1996). Bleichmar (1983), por exemplo, argumenta que uma pessoa pode ter uma visão pessimista do futuro, considerar sua vida um fracasso, contemplar a idéia de suicidar-se, manifestar idéias que ninguém vacilaria catalogar como claramente melancólicas, e, no entanto, não demonstrar tristeza, podendo predominar ao contrário raiva e irritação consigo mesmo. Em outros casos, o mesmo conjunto de idéias pode ocorrer com uma marcante frieza emocional, não existindo um correlato com as emoções.

Ainda segundo Bleichmar (1983) o núcleo da depressão como estado não pode ser buscado no pranto, na tristeza, ou na inibição psicomotora, pois todos podem faltar, mas sim no tipo de idéias comuns a todos os quadros nos quais pelo menos uma destas manifestações está presente. Essas idéias não necessariamente se referem aos temas que se queixam os depressivos e que aparecem nos tratados de Psiquiatria, como as idéias de ruína, de fracasso, de inferioridade, de culpa. Se estas idéias são capazes de produzir depressão é porque todas elas implicam uma representação que o sujeito faz da não exequibilidade de um desejo em que alcançaria um ideal, ou uma medida, com respeito ao qual se sente arruinado, fracassado, inferior, culpado.

Neste sentido, Estevão (1997) também salienta que a tristeza não pode ser um critério diagnóstico da depressão nem da melancolia, pois a primeira tem o centro no sofrimento afetivo, e a segunda no conjunto de condutas patológicas e alterações biológicas e psicológicas. Para o autor, os conceitos de humor e afetividade não são bem definidos e não servem como base para categorias diagnósticas. Segundo ele, afetividade não é função psicológica e sim qualidade de vivência. As cores, os sabores, as temperaturas e os afetos são qualidades de nossas funções, portanto a afetividade é a maneira pela qual os acontecimentos da vida nos afetam.

Para a psicanálise, encontram-se na obra de Melanie Klein (1982) as explicações mais esclarecedoras sobre a psicodinâmica da depressão quando descreve as origens da posição esquizoparanóide e posição depressiva. Delouya (2000), em sua obra sobre a depressão, retoma as concepções kleinianas e afirma que "a perda do objeto, separação e luto parecem constituir um eixo possível para o tema da depressão” (p.29). A depressão eclode com a consciência de ser separado da mãe, ou com a perda progressiva dela, na esteira do nascimento do sujeito - do eu - e o conseqüente investimento em si. Situa-se portanto, em torno da configuração do objeto total que vai de par com o nascimento do eu e com a preocupação de ter destruído a mãe sentida como progressivamente perdida. $\mathrm{O}$ afeto depressivo situa-se, então, nesse ponto central de transição, constitutivo do psiquismo, onde a abdicação narcísica, da onipotência e da fusão, se faz necessária em vista do reconhecimento do objeto total.

Diante da complexidade e variabilidade da sintomatologia e principalmente da dicotomia entre os sintomas afetivos e os sintomas ideativos ou cognitivos da depressão, o estudo da sensibilidade diagnóstica de um instrumento que privilegia a dinâmica afetiva e emocional do indivíduo parece valioso. Entretanto, a partir de uma ampla busca nas bases de dados, não se encontra nenhuma referência ao estudo específico de depressão no teste de Pfister.

O objetivo do presente trabalho é verificar os 


\section{Anna Elisa de Villemor Amaral}

alcances e limitações do teste das Pirâmides Coloridas de Pfister para o diagnóstico do quadro psicopatológico da depressão, considerando que a sintomatologia desse quadro é bastante complexa. Pretende-se identificar características quanto à freqüência das cores e ao aspecto formal das pirâmides que sejam significativas no grupo de pacientes quando comparado ao grupo de não pacientes.

\section{Método}

\section{Participantes}

Foram sujeitos desse estudo dezenove pacientes com depressão. Como critério de inclusão na amostra considerou-se o fato de atender aos requisitos do DSM-IV aferidos por meio da Entrevista Clínica Estruturada (SCID), revelando pelo menos um episódio depressivo. Embora cientes da variabilidade das sintomatologias, optamos por partir do diagnóstico nosográfico tal como é feito nas instituições de saúde, não diferençando os pacientes com base nas sintomatologias auto-relatadas. Todos os pacientes encontravam-se em tratamento psiquiátrico em clínicas de saúde mental da região. Não foram excluídas as co-morbidades uma vez que o interesse principal do estudo era a presença de depressão. O número mais restrito de participantes desse grupo resulta das dificuldades de se encontrar colaboradores ou de se dar continuidade na coleta de dados, uma vez que a mesma demandava cerca de duas horas no total.

Cento e dez indivíduos não-pacientes constituíram uma amostra normativa que serviu como base para comparação dos resultados. A amostra normativa foi composta por indivíduos voluntários que nunca apresentaram queixas específicas e que responderam a um questionário que visava obter dados de identificação, histórico de saúde e eventuais tratamentos (Villemor Amaral, Primi \& Silva, 2002).

\section{Material}

Foram utilizados dois instrumentos para a coleta de dados:

- A SCID: A SCID é um roteiro de entrevista semi estruturada para os critérios do DSM-IV, que foi usado para a confirmação dos diagnósticos psiquiátricos e uniformização dos critérios para composição do grupo experimental. Sua aplicação tem a duração de aproximadamente duas horas e possibilita a avaliação de aspectos ligados à identificação; vida escolar e profissional; início, evolução e história do tratamento da enfermidade; contexto ambiental; problemas atuais e funcionamento social atual. Verifica, detalhadamente, os sintomas e queixas apresentadas pelo paciente, permitindo uma conclusão diagnóstica.

Uma adaptação da revisão geral da SCID serviu como base para a entrevista com os indivíduos não-pacientes.

- O teste de Pfister. Consiste na execução de três pirâmides coloridas conforme o gosto do examinando. Assim, o material necessário para a aplicação compõe-se por um jogo de três cartões em papel pardo com o desenho do esquema de uma pirâmide, e um jogo de quadrículos coloridos formado por 10 cores subdivididas em 24 tonalidades. Esse jogo deve conter no mínimo de 40 a 45 quadrículos de cada tonalidade. O participante é convidado a construir suas pirâmides, uma de cada vez até totalizar o número de três, de modo que fiquem bonitas, do seu ponto de vista. Logo após é feito um breve inquérito, indagando-se sobre sua preferência quanto às pirâmides executadas e cores em geral. Para esse estudo os dados foram analisados levando-se em conta especificamente à freqüência das cores e o aspecto formal.

\section{Procedimentos}

Os indivíduos do grupo de pacientes foram selecionados nas instituições de saúde de uma região do interior de São Paulo com colaboração das mesmas após análise do comitê de ética de cada uma delas e da Universidade. Os pacientes eram inicialmente encaminhados pelos profissionais da instituição, com base nos diagnósticos prévios. Após esclarecimento dos objetivos e procedimentos da pesquisa o paciente era convidado a assinar o termo de consentimento e iniciava-se a aplicação da SCID. Em seguida, a aplicação do Pfister foi realizada de acordo com o proposto por Villemor Amaral (1978), tendo como instrução a construção de três pirâmides bonitas. As 
instituições disponibilizaram ambiente adequado para a aplicação do teste, garantindo certo conforto e privacidade.

O grupo de não-pacientes foi constituído também por indivíduos voluntários, selecionados em empresas da região, procurando-se uma composição semelhante ao grupo patológico quanto à faixa etária - entre 18 e 50 anos - e escolaridade - predominantemente primeiro grau. Todos foram esclarecidos quanto aos objetivos da pesquisa e também convidados a assinar o termo de consentimento. Passaram por uma entrevista mais reduzida, visando excluir problemas graves e confirmar a inexistência de busca por assistência psicológica ou psiquiátrica ao longo da vida e logo a seguir submetiam-se a aplicação do Pfister. O local de aplicação era a própria empre- sa onde trabalhavam, em local apropriado e com privacidade.

\section{Resultados e discussão}

Realizou-se um exame dos dados obtidos procedendo uma análise de regressão logística. Essa análise tinha por objetivo verificar quais, dentre as variáveis do Pfister selecionadas - freqüência das cores e aspecto formal, poderiam contribuir para o diagnóstico da depressão, investigando quais indicadores permitem prever a pertença de um paciente no grupo de depressão. Este procedimento está descrito de forma mais minuciosa em Villemor Amaral, AE; Primi, R e Silva T.C. (2002).

Nessa análise encontra-se como resultado significativo, do ponto de vista estatístico, o conjunto de dados apresentados na tabela 1.

Tabela 1: Resultado da análise de regressão logística

\begin{tabular}{|lcccccc|}
\hline Variável & B & Erro Padrão & Wald & gl & Sig & Exp (B) \\
& & & & & & \\
P_Vd & 0,055 & 0,025 & 4,714 & 1 & 0,030 & 1,056 \\
CA_VI & 1,088 & 0,640 & 2,889 & 1 & 0,089 & 2,967 \\
$\begin{array}{l}\text { Formações } \\
\text { cortadas }\end{array}$ & 2,0241 & 1,2701 & 2,5396 & 1 &, 1110. & 7,5694 \\
$\begin{array}{l}\text { Formações } \\
\text { tendendo a }\end{array}$ & & & & & & \\
estruturas & 9,268 & 25,111 & 0,136 & 1 & 0,712 & 10597,747 \\
Constant & $-3,922$ & 0,858 & 20,889 & 1 & 0,000 & 0,020 \\
\hline
\end{tabular}

P_Vd = porcentagem de verde

CA_VI = Constância absoluta de violeta

Na tabela 1 constata-se que foram a porcentagem da cor verde, a constância absoluta da cor violeta, as formações cortadas (pirâmides em que toda uma camada é composta pela cor branca) e as formações tendendo a estrutura os indicadores que se revelaram como significativos no grupo de pacientes com depressão quando comparados com os não-pacientes.

As coordenadas da curva de ROC, que demonstram a sensibilidade e a especificidade dos diagnósticos com base nesses indicadores, são apresentada na tabela 2 . 
Tabela 2: Coordenadas da curva de ROC

\begin{tabular}{|c|c|c|}
\hline $\begin{array}{c}\text { Diagnóstico } \\
\text { positivo se a } \\
\text { Probabilidade } \\
\text { for igual ou } \\
\text { maior que }\end{array}$ & $\begin{array}{c}\text { Sensibilidade } \\
\text { (Verdadeiros } \\
\text { Positivos) }\end{array}$ & $\begin{array}{l}1 \text {-Especificidade } \\
\text { (Falsos Positivos) }\end{array}$ \\
\hline 0000000 & 1,000 & 1,000 \\
\hline 0220300 & 947 & 982 \\
\hline 0261919 & 947 & ,973 \\
\hline 0294827 & 947 & 955 \\
\hline 0331728 & 947 & ,928 \\
\hline 0373070 & 947 & 874 \\
\hline 0419340 & ,947 & ,793 \\
\hline 0471066 & 947 & ,739 \\
\hline 0528820 & ,947 & 658 \\
\hline ,0565926 & 947 & ,613 \\
\hline 0599860 & 947 & ,604 \\
\hline 0664894 & 895 &, 577 \\
\hline 0741576 & ,895 &, 568 \\
\hline 0783485 & ,895 & ,559 \\
\hline ,0829603 & ,895 & ,532 \\
\hline 0876038 & ,895 & ,523 \\
\hline 0927029 & 842 &, 505 \\
\hline 0978363 & ,842 & 468 \\
\hline ,1034603 & ,842 & ,432 \\
\hline 1091211 & ,842 & ,396 \\
\hline ,1153070 & 842 & 342 \\
\hline 1215322 & ,789 & ,324 \\
\hline 1283156 & ,737 & 279 \\
\hline 1420738 & ,737 & ,225 \\
\hline 1510849 & ,579 & ,162 \\
\hline 1591593 & ,579 & 153 \\
\hline 1675664 & ,579 & ,144 \\
\hline ,1763252 & 579 & ,135 \\
\hline 1926149, & ,579 & ,090 \\
\hline 2027410 & ,526 & ,090 \\
\hline 2207222 & ,474 & ,090 \\
\hline 2418728 & 474 & 081 \\
\hline 2780575 & ,474 & ,072 \\
\hline ,3237290 & ,421 &, 054 \\
\hline 3509110 & ,368 & ,045 \\
\hline ,3841075 & ,316 & ,036 \\
\hline ,4182507 & ,263 & ,036 \\
\hline 4580679 & 263 & ,027 \\
\hline 4972633 & ,263 & ,018 \\
\hline ,5266196 & ,211 & 018 \\
\hline ,5578154 & ,158 & ,018 \\
\hline ,6029470 & , 158 & ,009 \\
\hline 6584684 & ,105 & 009 \\
\hline 8418180 & , 105 & ,000 \\
\hline ,9992060 & ,053 &, 000 \\
\hline 1,0000000 & ,000 & ,000 \\
\hline
\end{tabular}

Tais dados revelam que os indicadores apon- tados na tabela 1 podem ser considerados significativos e discriminantes de depressão com uma sensibilidade de $84,2 \%$ de diagnóstico e uma especificidade de $34,2 \%$. Ou seja, embora esses indicadores possam ser úteis para identificar verdadeiros pacientes com depressão (verdadeiros positivos), há o risco alto de se classificar como sendo deprimidos indivíduos que realmente não o são (falsos positivos) já que a especificidade é baixa.

Entre os indicadores que se esperava encontrar, como o aumento do preto, ou mesmo da cor violeta, nenhum apresentou resultados significativos pela análise de regressão logística. A primeira variável destacada como significativa foi o aumento da cor verde, o que contraria a hipótese inicial de que a mesma poderia estar rebaixada entre os pacientes deprimidos devido a sua significação de bom contato interpessoal. Entretanto, retomando o significado da cor verde, ve-se que somente valores na média correspondem a um bom contato com o ambiente e boa capacidade de relacionamento e que seu aumento indica estados propícios a elevação da ansiedade devido ao acúmulo ou sobrecarga de estimulação interna impregnada de emoções que sufocam o indivíduo, enfraquecendo e rompendo o controle homeostático (Villemor Amaral, 1978 ). Esse dado parece ser corroborado pela presença constante da cor violeta no grupo de pacientes que embora não tendo sua freqüência significativamente aumentada, revelou-se como cor de constância absoluta, ou seja, aparece nas três pirâmides feitas pelos pacientes, mesmo que sua freqüência permaneça dentro dos valores médios esperados. A cor violeta é, por excelência, uma cor que revela tensão e ansiedade. O violeta traz em si a excitação juntamente com a introversão e retenção dessa excitação. Segundo Villemor Amaral (1978), indivíduos que usam o violeta expressam desejo de alcançar algo que de algum modo pareça inacessível. Este dado é importante para os estudos visto que, segundo Bleichmar (1983), o conteúdo do pensamento do depressivo está fixado na representação de um desejo como algo irrealizável, o qual determina o retardo ou a quase anulação, da ideação, da percepção, da motilidade e das manifestações afetivas a que eles estão sujeitos. Também Lima (1999) comenta a existência de ansiedade geralmente associada à depressão. Isso permite com- 
preender como, do ponto de vista teórico, a constância absoluta de violeta associada ao aumento da cor verde pode ser significativa para a caracterização da depressão no Pfister.

No que concerne ao aspecto formal, foram obtidos valores significativos de formações cortadas ou decepadas - pirâmides nas quais o branco ou tonalidades esbranquiçadas forma uma camada da pirâmide - e de formações estratificadas tendendo a estruturas. O primeiro tipo revela um modo de formação reconhecido na literatura como característico de dificuldades de relacionamento e contato social embora tenham sido observados em casos com características esquizóides (Villemor Amaral, 1978). Exner (1998), quando estudava pacientes com depressão por meio de Rorschach, verificou a alta incidência de dificuldade de relacionamento e de enfrentamento de situações sociais entre os pacientes com queixas de depressão, o que o fez criar o índice de déficit relacional (CDI). Os resultados deste estudo vão de encontro a essas observações, sendo possível considerar as pirâmides cortadas como indicadores desse déficit relacional.

O segundo tipo de aspecto formal que aparece como significativo foi o das formações que tendem à estruturas. É interessante notar que essas formações correspondem a um nível médio de maturidade emocional em vias de melhor estruturação. Se considera que a depressão, do ponto de vista psicanalítico, tem suas origens em um estágio mais evoluído do desenvolvimento que envolve a integração e a percepção do objeto total (Delouya, 2000; Melanie Klein, 1982), em contraposição à visão cindida e fragmentada típica dos estados mais primitivos da mente, vê-se que o resultado é coerente com maior nível de integração e estruturação do eu, quando comparados com pacientes mais regredidos, embora não atingindo os estágios mais diferenciados de amadurecimento representados pelas estruturas.

Conforme verifica-se na literatura, a sintomatologia da depressão é bastante variada, por vezes prevalecendo aspectos cognitivos e em outras prevalecendo aspectos sociais ou afetivos. Na composição da amostra, não distingue-se o tipo de sintomatologia prevalente em cada paciente, mas mesmo assim, pode-se constatar que tanto características cognitivas mais intimamente relacionadas com os níveis de diferenciação da forma, quanto aspectos sociais e afetivos estão, até certo ponto, representados nos indicadores que se revelaram estatisticamente significativos nesse estudo.

\section{Conclusão}

Os resultados obtidos com o teste de Pfister são compatíveis com descrições de alguns sintomas e dinamismos característicos das depressões. Mostrou-se significativo o aumento do verde acompanhado da constância absoluta da cor violeta, bem como a maior incidência de pirâmides cortadas e tendendo a estruturas. A combinação desses indicadores aponta para uma probabilidade de $84,2 \%$ para se identificar pacientes com depressão. Tais resultados permitem afirmar que o teste das Pirâmides Coloridas de Pfister pode colaborar para o diagnóstico de depressão dentro dos referidos limites de sensibilidade e especificidade, sendo contudo necessários outros recursos para maior segurança diagnóstica.

Por outro lado, é importante destacar, que ainda que a precisão dos indicadores para diagnóstico nosográfico da depressão por meio do Pfister não seja ideal, havendo ainda necessidade de mais estudos, pode-se dizer que sua utilidade reside na contribuição dada para a compreensão dos aspectos dinâmicos idiográficos de cada paciente que, associados a outros dados, podem ampliar a compreensão que se tem sobre o indivíduo.

\section{Referências Bibliográficas}

Associação Psiquiátrica Americana (1994). Manual diagnóstico e estatístico de transtornos mentais - DSM-IV. Porto Alegre: Artes Médicas.

Bauer, J.F. (1979). A estrutura em tapetes no teste das Pirâmides Coloridas de Pfister. Dissertação de mestrado. Universidade de São Paulo.

Berlinck, M.T. \& Fedida, P. (2000). A clínica da depressão: questões atuais. Rev. Latinoamericana de Psicopatologia Fundamental, III(2), 9-25.

Bleichmar H. (1983). Depressão: um estudo psicanalítico. Porto Alegre: Artes Médicas.

Carnio, E.C. \& Loureiro, S.R. (1993). Caracteriza- 
176 Anna Elisa de Villemor Amaral

ções da percepção real, de pacientes esquizofrênicos, avaliados através das técnicas das Pirâmides Coloridas de Pfister. Psico, 24(1) 35-47.

Cunha, J. A., Prieb, R.G.G., Goulart, P.M. \& Lemes, R.B. (1996). O uso do inventário de Beck para avaliar a depressão em universitários da PUCRS. Psico, 27(1), 106-115.

Delouya, D. (2000). Depressão. São Paulo: Casa do Psicólogo.

Estevão, G. (1997). Do Diagnóstico da depressão e suas implicações terapêuticas. Temas: Teoria e prática de psiquiatria,27(53), 71-84.

Exner, J.E. (1998). El Rorschach. Un Sistema Comprehensivo. Volume 1: Fundamentos Básicos. Madrid: Psimática.

Gauer, G.J.C. (1998). Atividade Natural Killer em pacientes com depressão maior. Rev. psiquiátrica, 20(3), 48-55.

Klein, M. (1982). Contribuições à Psicanálise. Uma contribuição à psicogênese dos estados maníacos-depressivos. São Paulo: Mestre Jou.

Lima, M.S. (1999). Epidemiologia e impacto social. Revista Brasileira de Psiquiatri, 21 (supl. 1): SI1-SI5.

Nuber, U. (1991). Depressão a doença mal compreendida. São Paulo: Pensamento.

Oliveira, E.A., Pasian, S.R. \& Jacquemin, A. (2001). A vivência afetiva em idosos. Psicologia Ciência e Profissão,21(1), p.68 - 83.

Rodrigues, M.J.S.F. (2000). O diagnóstico de Depressão. Psicologia USP, 11(1), 155-187.

Rorschach, H. (1972). Psicodiagnóstico. São Paulo: Mestre Jou.

Smith, V.H. (1999). Enfrentamento na depressão: Estilo Explanatório e tipos de experiência depressiva. Revista Estudos de Psicologia,16(3), 19-31.

Villemor Amaral, F. (1978). Pirâmides Coloridas de Pfister. Rio de Janeiro: CEPA. $2^{a}$ edição.
Villemor Amaral, A.E., Primi, R. \& Silva, T.C. (2002). O Teste das Pirâmides Coloridas de Pfister e o Transtorno Obsessivo Compulsivo. Avaliação Psicológica, (2), 133139. 